ARTICLE

Received 12 Jul 2012 | Accepted 5 Nov 2012 | Published 4 Dec $2012 \quad$ DOl: 10.1038/ncomms2256

\title{
A fast and accurate SNP detection algorithm for next-generation sequencing data
}

Feng $\mathrm{Xu}^{1,2, \star}$, Weixin Wang ${ }^{1,2, \star}$, Panwen Wang ${ }^{1,2}$, Mulin Jun $\mathrm{Li}^{1,2}$, Pak Chung Sham ${ }^{3,4,5}$ \& Junwen Wang ${ }^{1,2,3,6}$

Various methods have been developed for calling single-nucleotide polymorphisms from next-generation sequencing data. However, for satisfactory performance, most of these methods require expensive high-depth sequencing. Here, we propose a fast and accurate single-nucleotide polymorphism detection program that uses a binomial distribution-based algorithm and a mutation probability. We extensively assess this program on normal and cancer next-generation sequencing data from The Cancer Genome Atlas project and pooled data from the 1,000 Genomes Project. We also compare the performance of several stateof-the-art programs for single-nucleotide polymorphism calling and evaluate their pros and cons. We demonstrate that our program is a fast and highly accurate single-nucleotide polymorphism detection method, particularly when the sequence depth is low. The program can finish single-nucleotide polymorphism calling within four hours for 10-fold human genome next-generation sequencing data (30 gigabases) on a standard desktop computer.

\footnotetext{
${ }^{1}$ Department of Biochemistry, LKS Faculty of Medicine, The University of Hong Kong, Hong Kong, China. ${ }^{2}$ Shenzhen Institute of Research and Innovation The University of Hong Kong, Shenzhen, China. ${ }^{3}$ Centre for Genomic Sciences, LKS Faculty of Medicine, The University of Hong Kong, Hong Kong, China. ${ }^{4}$ Department of Psychiatry, LKS Faculty of Medicine, The University of Hong Kong, Hong Kong, China. ${ }^{5}$ State Key Laboratory in Cognitive and Brain Sciences, The University of Hong Kong, Hong Kong, China. ${ }^{6} \mathrm{HKU}-\mathrm{BGI}$ Bioinformatics Algorithms and Core Technology Research Laboratory, The University of Hong Kong, Hong Kong, China. * These authors contributed equally to this work. Correspondence and requests for materials should be addressed to J.W. (email: junwen@hku.hk).
} 
$\mathrm{D}$ etecting genetic variations in human genome is vital to understanding the causes of phenotypic variations, including susceptibilities to cancers and infectious diseases. Single-nucleotide polymorphisms (SNPs) are one of the most common types of genetic variation in humans. SNPs have been reported to influence protein coding ${ }^{1}$, transcriptional regulation ${ }^{2}$, alternative splicing ${ }^{3}$ and non-coding RNA regulation ${ }^{4}$. A large number of SNPs has been identified in the Human Genome Project, ${ }^{5,6}$ and the Human Haplotype Map Project ${ }^{7,8}$. In addition, recent advances in next-generation sequencing (NGS) technologies have enabled us to detect even more SNPs. The use of NGS platforms, such as the Illumina Genome Analyzer, Roche/454 FLX and ABI SOLiD, not only increases the throughput of data but also dramatically reduces the cost of sequencing 9 . Although SNP detection methods for conventional sequencing technologies are well developed, new SNP detection methods for NGS technologies are still lacking.

Several methods have been developed for SNP calling from NGS data, and the performances of these SNP calling programs have been evaluated ${ }^{10}$. For example, the SNP calling method by Morin et al. ${ }^{11}$ used a proportion of bases that matched the reference. However, this method used an arbitrary threshold and did not provide confidence estimates for the predicted results. Other methods such as MAQ ${ }^{12}$ and SOAPsnp ${ }^{13,14}$ are based on Bayesian-based posterior probabilities, and $\mathrm{SNVmix}^{15}$ uses a mixed binomial model to discover SNPs, giving a confidence score for each SNP called. These methods perform better for loci with high sequence depths, but their accuracy decreases for sequence depths lower than 10. Other SNP callers used in NGS analysis are integrated into pipelines or are structured into software libraries, such as Bcftools ${ }^{16}$ in samtools and UnifiedGenotyper in GATK ${ }^{17}$. Both of these tools use Bayesian likelihood to infer the posterior probability of a locus being a SNP and to call the genotype. Furthermore, both methods can use pooled data to improve the accuracy of the SNP calling. A recent software tool ${ }^{18}$ was developed for SNP calling of lower sequencing depths. However, the SNP filtering parameters were manually defined and the program accepted inputs from only the Solexa platform. This software performed better when known SNPs of the target genome were available.

We have designed a fast and accurate SNP detection (FaSD) program that uses a binomial distribution-based algorithm and a mutation probability to detect SNPs from NGS data. We compared our method with existing software using both cancer and normal tissue data from The Cancer Genome Atlas $(\text { TCGA })^{19}$ and trios data from 1,000 Genomes Project ${ }^{20}$. Using SNP arrays and high-depth sequencing data as benchmarks, we found that our method had higher SNP calling accuracy compared with other methods, especially with low-depth sequencing data. Furthermore, our program completed the SNP calling from 10 -fold human genome NGS data (30 gigabases) within four hours on a standard desktop computer compared with the GATK method that takes double the time.

\section{Results}

Performance evaluation on SNPs covered by arrays. To assess the SNP calling quality of the tools, we compared the results from our FaSD method with GATK ${ }^{17}$, SOAPsnp ${ }^{13,14}$, MAQ ${ }^{12}$, SNVmix2 (ref. 15) and Bcftools ${ }^{16}$ using data sets derived from a Glioblastoma multiforme (GBM) tumour sample and the corresponding blood normal sample from the same individual, which were both sequenced on a Illumina Genome Analyzer II platform. We used genotype calling results from both Affymetrix and Illumina SNP arrays as gold standards, which were obtained from the same samples. Because of the poor accuracy of SNP calling for data with very low sequencing depths ${ }^{12,13}$, we included only the loci that were covered by at least four reads. We compared the genotype concordances ${ }^{17}$ (Supplementary Table S1) among the SNP calling tools and the two SNP arrays for both normal and tumour data sets. Looking at the normal data set with either Bowtie (Table 1) or BWA (Supplementary Table S2) as the aligner, the genotypes called by both Affymetrix SNP array 6.0 and Illumina humanhap550 genotyping beadchip array were very similar with concordance rates of more than 0.95 ( 0.997 for bowtie and 0.957 for BWA). SOAPsnp and MAQ, both developed by the Beijing Genome Institute in Shenzhen, China, also showed high concordances of 0.997 with either Bowtie or BWA as the aligner. The genotypes called by GATK and Bcftools were very similar when using Bowtie as the aligner giving a concordance of 0.979 . The concordance drops to 0.924 when BWA was used as the aligner, but this value was still high compared with other genotype calling methods. By comparing the results from the SNP calling programs with those from the two arrays used as our gold standards, we found that FaSD and Bcftools were the best methods. FaSD was better than Bcftools when Illumina array was used as the benchmark (Table 1 and Supplementary Table S2), as shown by a concordance of 0.882 for FaSD vs. 0.865 for Bcftools when aligned by Bowtie, and 0.833 vs. 0.674 when aligned by BWA. We also evaluated the performance of these methods on the tumour data set, because tumour tissues are highly heterogeneous compared with normal tissue. With either Bowtie or BWA as the aligner, FaSD showed a higher concordance of about 3-5\% with benchmarks in the tumour tissue compared with normal tissue (Table 1 and Supplementary Table S2). Similar concordance increases were observed in Bcftools with both Bowtie and BWA, but this was not the case for others. In contrast, MAQ and SOAPsnp showed a slight drop in concordance for tumour tissue compared with normal tissue.

The area under the curve (AUC) of a receiver operating characteristic (ROC) curve is widely used as a measure of the overall classification performance of a program without needing

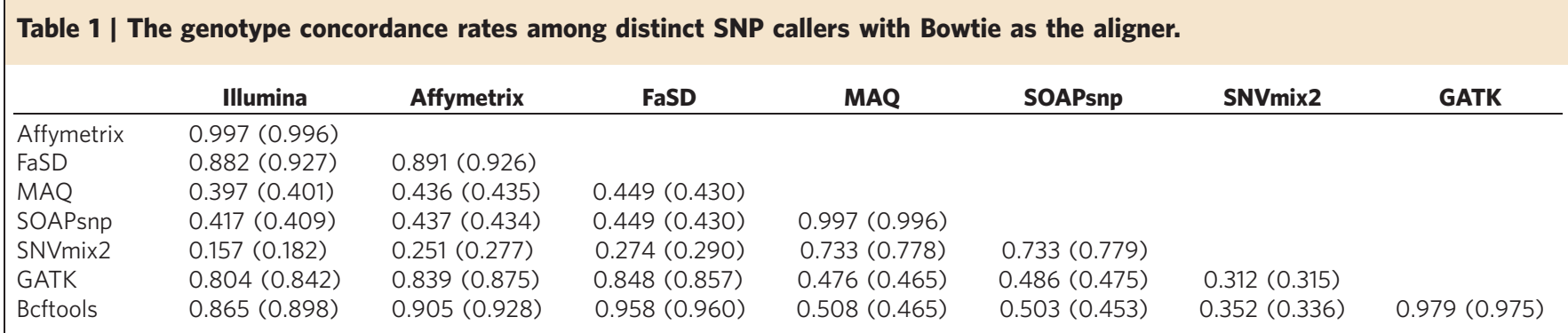

The first number in each cell is the concordance between corresponding SNP callers in the normal data sets, the number in the parentheses is the concordance in the tumour data sets. The average depth of both normal and tumour data sets was $10 \times$ 
to consider the specific cutoffs. We independently evaluated the performances by comparing the AUCs of different programs using both normal and tumour data sets. Because the sequencing depth has a large impact on SNP calling quality, we separated all the data sets into four sub-data sets according to the sequencing depth of each position. The subsets were named 4_5, 6_10,11_15 and $16 \_20$, which corresponded to sequence depths of $4-5,6-10$, 11-15 and 16-20, respectively. As shown in Supplementary Tables S3 and S4, FaSD had the largest AUC compared with the other tools for all the sub-data sets of both the tumour and normal data sets, regardless of the array platform used as the benchmark. We further tested the performances by the stability of AUCs (bootstrapped 1,000 times), and the result confirmed that FaSD significantly outperformed the other methods, especially in low coverage categories (Fig. 1, one tail unpaired Wilcox test $P<2.2 \mathrm{e}-16$ for each depth category, in both GBM tumour and normal data sets, benchmarked by either Illumina or Affymetrix array).

We compared the results from FaSD, GATK, Bcftools and MAQ on both normal and tumour data sets. For the tumour samples using Affymetrix SNP array as the benchmark, SNPs were divided into several groups: SNPs detected by a single tool, two tools, three tools and by all four tools (Fig. 2). For a SNP called by FaSD and by either Bcftools or GATK, over $99.7 \%$ could be confirmed by the Affymetrix array. However, if a SNP was called by both Bcftools and GATK, but not by FaSD, only $46.2 \%$ could be confirmed by the Affymetrix array. We further looked at the SNPs that were called uniquely by each tool: $63.3 \%$ of SNPs detected by only FaSD could be confirmed by the Affymetrix

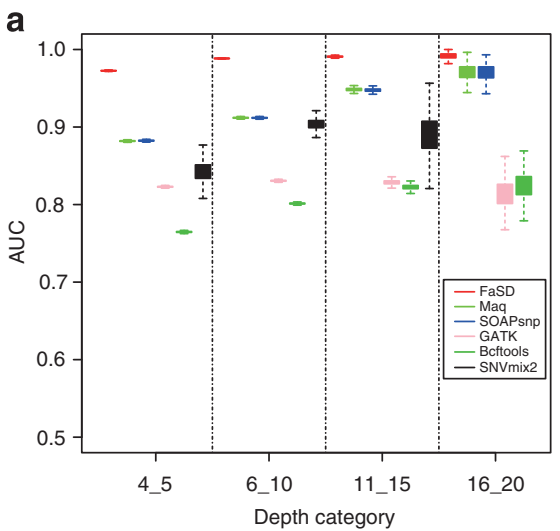

C

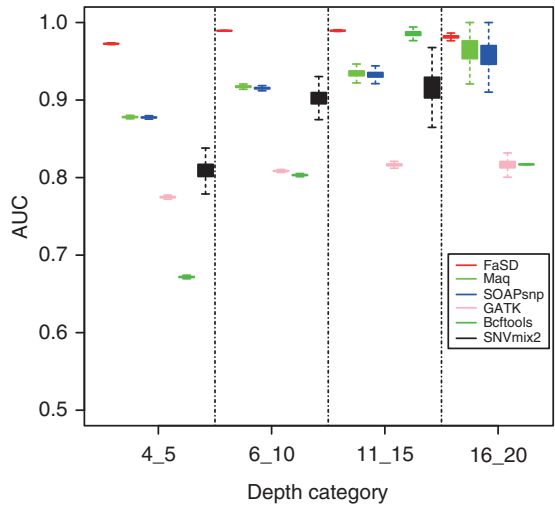

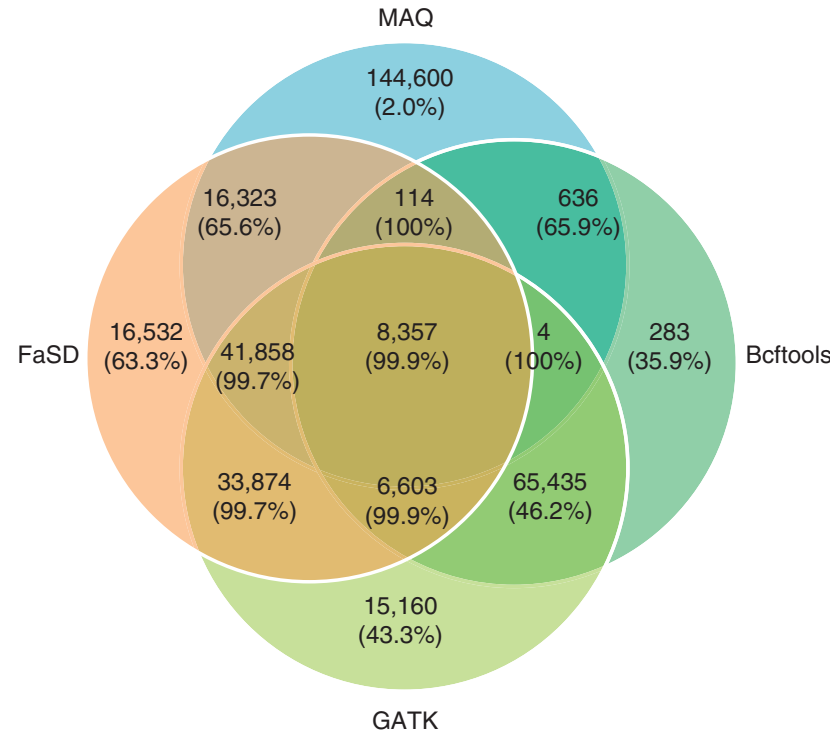

Figure 2 | The Venn diagram of SNPs detected by different tools. The number in each cell is the number of SNPs in the corresponding category. The percentage under the number is the proportion of SNPs that were confirmed by the Affymetrix SNP array. The FaSD, GATK, Bcftools and MAQ called 123661, 171291, 81432 and 211892 SNPs in total, respectively. The average depth of this data set was $10 \times$. The figure is based on the tumour data set and Bowtie was used as aligner, and statistics are based on the loci genotyped by the Affymetrix SNP array.

b

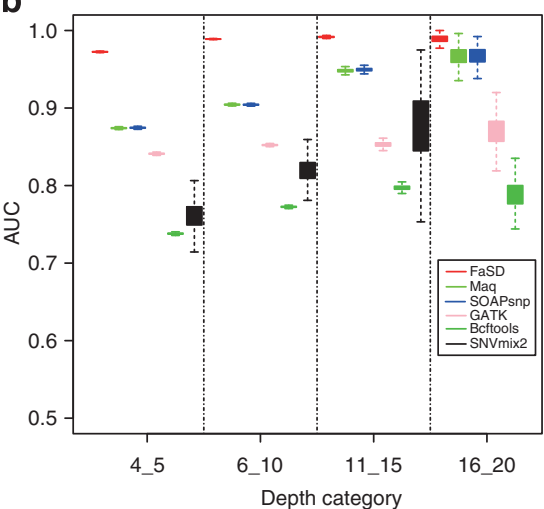

d

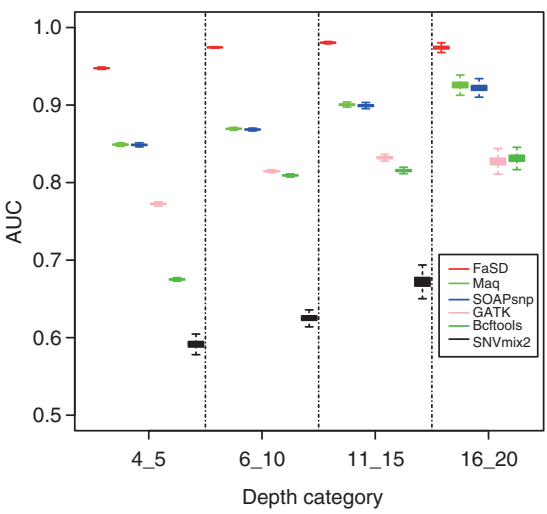

Figure 1 | Box plots of the AUC of each program based on 1,000 times bootstrap. The average depth of this data set was $10 \times$. (a) The normal data set with Affymetrix SNP array as the benchmark, (b) normal data set with Illumina SNP array as the benchmark, (c) tumour data set with Affymetrix SNP array as the benchmark and (d) tumour data set with Illumina SNP array as the benchmark. The average sequencing depth in both normal and tumour data sets was $10 \times$. The number of loci located in the 16_20 category was less than the number of loci located in the other categories. This explains why some tools in 16_20 category showed a small decrease in the AUC with a corresponding slight increase in the standard deviation compared with the other categories. 
array, whereas the number was $43.3 \%$ for GATK, $35.9 \%$ for Bcftools and $2.0 \%$ for MAQ. MAQ made the largest number of SNP calls, but the accuracy was the lowest for uniquely called SNPs. We analysed the normal data set and found similar results (Supplementary Fig. S1).

Performance evaluation on SNPs not covered by arrays. Both Illumina and Affymetrix SNP arrays contain less than one-tenth of the total number of current SNPs in the human genome, so evaluations based on the SNP arrays will be biased for common SNPs selected by these platforms. To avoid this bias, we evaluated the performance of the tools using high coverage multi-sourcemerged sequencing data (chromosomes 21 and 22 from a Yoruba with average 35 -fold whole genome coverage). Because this data set has high sequence depth and MAQ has been proven to have high SNP calling accuracy for high sequence depth data ${ }^{12}$, we used the results of MAQ SNP calling (named High_MAQ) on this data set as our benchmark. We randomly sampled $10 \%$ of reads four times to form four sub-data sets. FaSD, MAQ, SOAPsnp, GATK and Bcftools were then used to call SNPs on these subdata sets and their results were compared with the benchmark. The majority of loci $(>99.5 \%)$ in the human genome have the genotype of AA, whose inclusion could overwhelm any differences, that is, the overall genotype concordance won't differ much among the various caller methods. Therefore, we used non-reference concordances to assess the quality of SNP calling by excluding the concordant AA genotypes (Supplementary Table S1). As shown in Table 2, with default cutoffs, FaSD called 69,768 SNPs in chromosome 21 and 78,240 SNPs in chromosome 22. The numbers are close to the SNPs called by the benchmark, which were 78,679 and 68,017 , respectively. SOAPsnp and MAQ called 25-40\% more SNPs than the benchmark, whereas Bcftools and GATK called 40-50\% less SNPs than the benchmark. To call a similar number of SNPs as Bcftools and GATK, we adjusted FaSD's cutoff from the default 3.2 to 5.0, which reduced numbers of SNPs called in chromosomes 21 and 22 to 45,845 and 45,211, respectively. We then compared the non-reference concordances of FaSD at this cutoff with the other tools and with the benchmark. For chromosome 21, FaSD had the highest non-reference concordance with the benchmark (Table 3). Consistent with our previous evaluation, the performance of SOAPsnp was almost the same as $\mathrm{MAQ}^{10}$. The non-reference concordances of both GATK and Bcftools with the benchmark were also around 0.4, which was close to that of FaSD. For chromosome 22, GATK had the best non-reference concordance with the benchmark. However, the non-reference concordances of both FaSD and Bcftools were only around $0.5 \%$ and $2.5 \%$ lower than GATK, respectively (Supplementary Table S5).

Performance evaluation on pooled data. The detection of rare variants is important because common genetic variants can explain only a small proportion of heritability ${ }^{21}$. However, rare variants have low minor allele frequencies and are very hard to separate from genotype errors ${ }^{22}$. Pooled data from multiple individuals can improve the discovery of rare variants. Both GATK and Bcftools have the function to utilize pooled data. Therefore, we compared the performance of FaSD on pooled samples with the performance of both GATK and Bcftools. The pooled samples are composed of low coverage $(\sim 4 \times)$ whole genome sequencing data of $40 \mathrm{CEU}$ (Utah residents with ancestry from Northern and Western Europe) individuals from pilot 1 of 1,000 Genomes Project. Sequencing data on chromosome 21 of three CEU individuals in one trio were picked as the evaluation objects. MAQ calling results of corresponding high coverage data $(30 \times)$ from pilot 2 of 1,000 Genomes Project were used as the gold standard. As expected, the calling results on the individual's low coverage $4 \times$ data had limited SNPs discovery. Even using FaSD as the caller, we could not exceed the non-reference concordance of 0.6 (Table 4 and Supplementary Tables S6 and S7). Using a multi-sample SNPs calling function to genotype

Table 4 | The non-reference concordances for chromosome 21 in individual call set and pooled-sample call set of NA12878.

\begin{tabular}{lccc} 
& High_MAQ & FaSD & GATK \\
\hline FaSD & $0.557(0.556)$ & & \\
GATK & $0.489(0.379)$ & $0.637(0.641)$ & \\
Bcftools & $0.535(0.353)$ & $0.573(0.673)$ & $0.520(0.603)$
\end{tabular}

The first number in each cell is the non-reference concordance on the basis of pooled data, the number in the parentheses is the non-reference concordance based on the corresponding individual low coverage data set. High_MAQ was used as the benchmark.

Table 2 | Number of reported SNPs in each method for chromosomes 21 and 22.

\begin{tabular}{lcccccc} 
Data set software & High_MAQ & Bcftools & GATK & FaSD & MAQ & SOAPsnp \\
\hline 21 & 78,679 & 39,688 & 48,136 & 69,768 & 97,666 & 97,267 \\
22 & 68,017 & 33,028 & 36,867 & 78,240 & 94,237 & 94,768 \\
\hline
\end{tabular}

The total number of SNPs called by each software using default settings. High_MAQ was the SNP calling result from high-depth data, which was used as our benchmark. The lengths of chromosomes 21 and 22 are $46,976,537$ and $49,476,972$, respectively; the GC\% was $43 \%$ and $49 \%$, respectively. The average depth was $4 \times$ for chromosome 21 and 22 .

Table 3 | The non-reference concordances for chromosome 21.

\begin{tabular}{|c|c|c|c|c|c|}
\hline & High_MAQ & FaSD & MAQ & SOAPsnp & GATK \\
\hline $\mathrm{FaSD}$ & $0.419 \pm 0.002$ & & & & \\
\hline MAQ & $0.271 \pm 0.001$ & $0.267 \pm 0.001$ & & & \\
\hline SOAPsnp & $0.266 \pm 0.001$ & $0.264 \pm 0.001$ & $0.981 \pm 0.001$ & & \\
\hline GATK & $0.415 \pm 0.001$ & $0.626 \pm 0.002$ & $0.315 \pm 0.001$ & $0.308 \pm 0.001$ & \\
\hline Bcftools & $0.383 \pm 0.001$ & $0.613 \pm 0.002$ & $0.295 \pm 0.001$ & $0.293 \pm 0.001$ & $0.681 \pm 0.002$ \\
\hline
\end{tabular}


multiple samples simultaneously from aggregated 40-sample data, GATK and Bcftools showed at least 20\% improvement in terms of the non-reference concordance. FaSD also showed a slight increase in the non-reference concordance because it also incorporated genotype information from the pooled data sets. For all individuals investigated from that trio, FaSD had the best non-reference concordances of 0.557 (NA12878), 0.585 (NA12891) and 0.556 (NA12892) benchmarked by High_MAQ. In the GATK pipeline, imputation ${ }^{23,24}$ could help to refine and recover genotypes at sites with little or no coverage. Following this recommendation, we used Beagle ${ }^{25}$ to impute the 40 -sample call set. It should be noted that imputation not only recovered an additional $36 \%$ of the non-reference sites for FaSD, $26 \%$ for GATK and 3\% for Bcftools (average of three individuals, Supplementary Table S8), but also improved the non-reference concordance from an average of 0.566 to an average of 0.706 for FaSD, from 0.477 to 0.632 for GATK and from 0.514 to 0.590 for Bcftools (average of three individuals, Supplementary Tables S9-11).

Processing speed. The time taken for these tools to process the data is a major bottleneck for NGS data analysis. We compared the running time of FaSD, GATK and Bcftools for SNP calling on a standard 10-fold tumour genome (total 30 gigabases) NGS data. All three programs were tested on a server (based on a $2.13-\mathrm{GHz}$ Intel Xeon Processor E5506 CPU with $4 \mathrm{MB}$ cache, $32 \mathrm{~GB}$ memory and $4 \mathrm{~TB}$ storage) and on a standard personal computer (running a 2.66-GHz Intel Core2 Quad Processor Q9400 CPU with $6 \mathrm{MB}$ cache, $6 \mathrm{~GB}$ memory and $1 \mathrm{~TB}$ storage). On the server using only a single core, GATK took 29,757 s to finish the job and Bcftools took 19,286 s, whereas FaSD took only 13,484 s, which was $120 \%$ faster than GATK and $43 \%$ faster than Bcftools (Fig. 3).

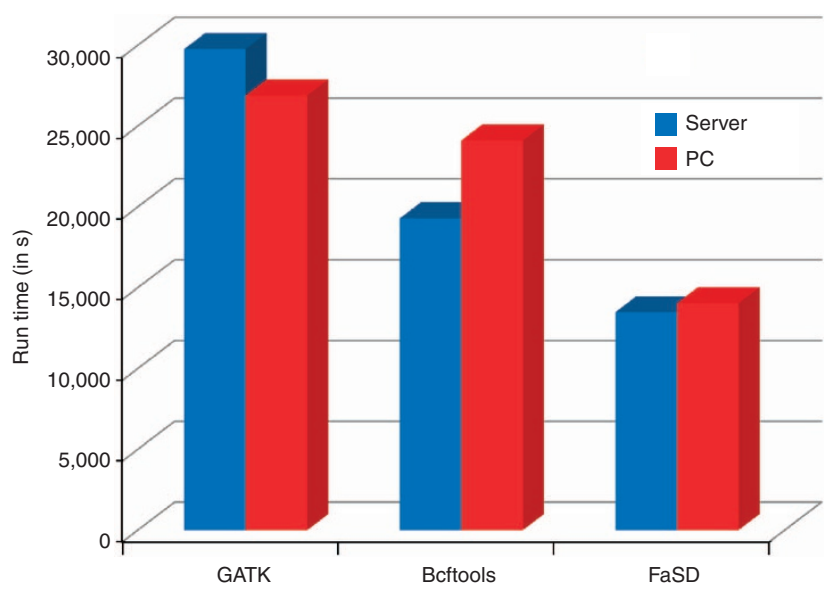

Figure 3 | Run time on both server and standard personal computer. The average depth of this tumour data set was $10 \times$ (30 gigabases). Bowtie is applied as aligner.
On the personal computer, FaSD finished the job in 14,042 s, which was $92 \%$ faster than GATK and $72 \%$ faster than Bcftools.

\section{Discussion}

Our FaSD method could rapidly call SNPs after the NGS data are aligned to the reference genome. FaSD uses a single score (FaSD_score) to determine the loci's genotype. For all the experiments, we used a default cutoff of 3.2 for separating AA and $\mathrm{AB}$, and a cutoff of 15.8 for separating $\mathrm{AB}$ and $\mathrm{BB}$. Users can adjust these cutoffs in our program to obtain different numbers of SNPs depending on their purpose. As shown in Supplementary Table S12, a higher cutoff will result in less SNPs being reported, and vice versa. If the default cutoff of 3.2 is applied, the false positive rate and true positive rate are 0.0011 and 0.83 in the normal data set and 0.0015 and 0.82 for the tumour data set, respectively. To assess the AUC of each SNP caller, we assigned a SNP locus as class 1 , and a non-SNP locus as class 0 . The $0 / 1$ ratio is the ratio between the numbers of loci in class 0 and class 1 . The loci were determined to be truly class 0 or class 1 based on the gold standard used. An imbalanced data set could reduce the classification performance and make the classifications deviate to the prevalent class ${ }^{26,27}$. The $0 / 1$ ratios of both the normal data set and tumour data set were close to 1 (Supplementary Table S13), indicating little classification bias in our array-based data sets.

Our FaSD algorithm is comprised of two parts, the alternative_score and the mutation probability (equations 1 and 2 in the methods section). The mutation probability model has been used in many other SNP detection programs. We report here for the first time the use of our unique alternative_score model. To assess the contributions of these two models to the overall performance of the FaSD method, we evaluated the AUCs separately and in combination from the normal data set benchmarked by the Affymetrix array. In the $4 \_5$ depth category (Table 5), the combined model had an AUC of 0.973, which decreased by 0.033 to 0.940 when only the mutation probability model was used but only decreased by 0.003 to 0.970 when only the alternative_score model was used, indicating the alternative_ score model contributed about $90 \%$ to FaSD's performance. At high depth, the AUC decreased by 0.009 using only the mutation probability model but decreased by 0.001 using only the alternative_score model, indicating the alternative_score contributed about $70-80 \%$ to FaSD's performance. Similar results were obtained when the two components were evaluated using the Illumina array (Table 5).

To assess the effects of different aligners on FaSD's performance, we used BWA as alternative aligner for the tumour and normal data set (BWA is the recommended aligner for GATK). On the basis of concordance, we showed that FaSD was the superior method (Supplementary Table S2). Although with BWA the AUC of GATK increases by about $10 \%$ in each sub-category, FaSD still has the largest AUCs (Supplementary Table S14), indicating FaSD's superior performance regardless of the aligner.

Table 5 | AUC of different parts of FaSD on the normal data set.

\begin{tabular}{|c|c|c|c|c|c|c|c|c|}
\hline \multirow[b]{2}{*}{ Depth } & \multicolumn{4}{|c|}{ Affymetrix } & \multicolumn{4}{|c|}{ Illumina } \\
\hline & 4_5 & 6_10 & 11_15 & 16_20 & 4_5 & 6_10 & 11_15 & 16_20 \\
\hline Mutation_probability & 0.940 & 0.958 & 0.976 & 0.983 & 0.937 & 0.953 & 0.961 & 0.979 \\
\hline Alternative_score & 0.970 & 0.988 & 0.990 & 0.989 & 0.967 & 0.988 & 0.990 & 0.986 \\
\hline FaSD (combined) & 0.973 & 0.981 & 0.992 & 0.992 & 0.972 & 0.989 & 0.992 & 0.989 \\
\hline
\end{tabular}


a

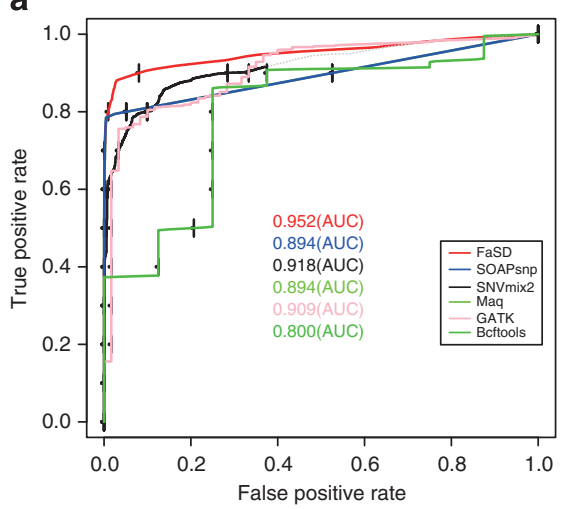

C

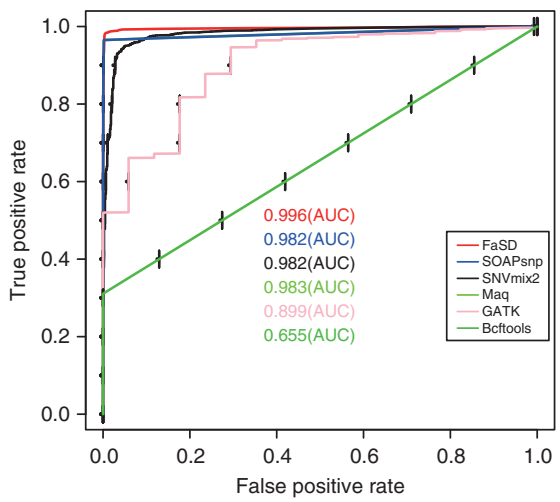

b

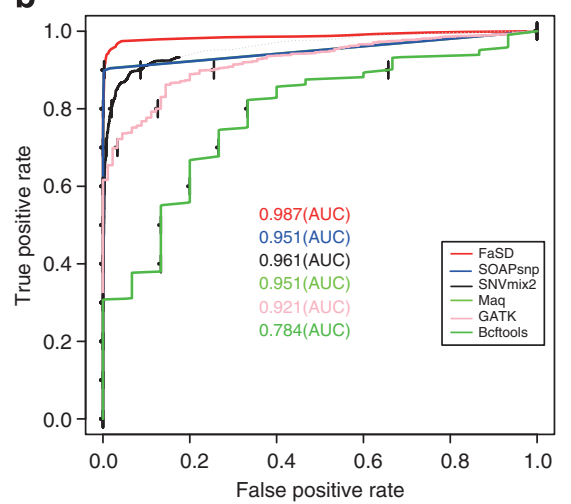

d

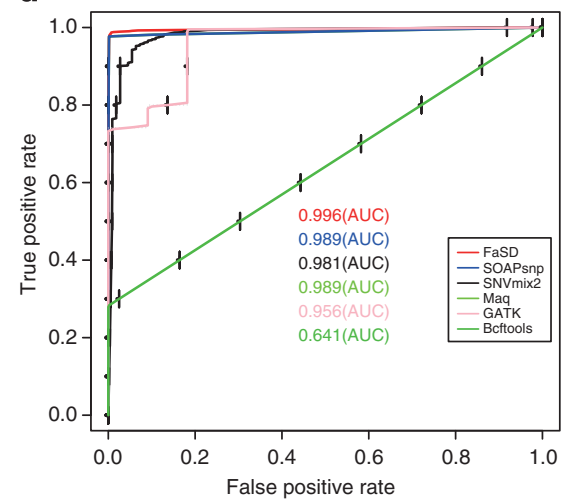

Figure 4 | The performance on the OV data set sequenced from the SOLiD sequencing platform. (a) The AUC on the $4 \_5$ coverage subset, (b) the AUC on the 6_10 coverage subset, (c) the AUC on the 11_15 coverage subset and (d) the AUC on the 16_20 coverage subset. The average depth of this data set was $6 \times$.

Table 6 | The concordance rates after realignment among distinct SNP callers.

\begin{tabular}{llllll} 
& Illumina & Affymetrix & FaSD & MAQ & SOAPsnp \\
\hline Affymetrix & $0.949(0.942)$ & & & & \\
FaSD & $0.864(0.867)$ & $0.922(0.929)$ & & & \\
MAQ & $0.439(0.469)$ & $0.454(0.486)$ & $0.484(0.491)$ & & \\
SOAPsnp & $0.439(0.472)$ & $0.455(0.488)$ & $0.485(0.492)$ & $0.998(0.995)$ & $0.532(0.564)$ \\
GATK & $0.790(0.774)$ & $0.912(0.930)$ & $0.950(0.962)$ & $0.531(0.561)$ & $0.534(0.564)$ \\
Bcftools & $0.735(0.734)$ & $0.898(0.930)$ & $0.951(0.958)$ & $0.534(0.562)$ & $0.980(0.978)$ \\
\hline
\end{tabular}

The first number in each cell is the concordance between corresponding SNP callers in the normal data set, the number in the parentheses is the concordance in the tumour data set. Bowtie was used as aligner. The average depth of both normal and tumour data sets was $10 \times$.

We then assessed the effects of the sequencing platform on FaSD's performance. We used the six tools to call SNPs from data sequenced using ABI-SOLiD sequencing platform. With the Affymetrix SNP array as the gold standard, FaSD outperforms all the other programs using the corresponding Serous Cystadenocarcinoma (OV) data set (One tail paired Wilcoxon test $P$-value $<2.2 \mathrm{e}-16$ in each depth category, Fig. 4 and Supplementary Fig. S2). This result confirmed that FaSD SNP calling is reliable even for data from different sequencing platforms.

The developers of GATK proposed a unified analytic framework that included realigning the sequences before SNP calling to reduce the effects of inaccurate base quality scores and mapping errors $^{23}$. We adopted this framework for locally realigning regions around indels and for recalibrating the base quality score to check how the pre-process affected the SNP calling quality. Realignment had significant effects on GATK's performance, particularly when using the Affymetrix array as the gold standard. Realignment caused GATK's concordance rates with the Affymetrix array to increase from 0.839 to 0.912 for normal tissue and increase from 0.875 to 0.930 for tumour tissue. Changes in FaSD performance resulted in a moderate concordance increase from 0.891 to 0.922 for normal tissue and an insignificant increase from 0.926 to 0.929 for tumour tissue (Tables 1 and 6). On the other hand, realignment with Illumina Array as the gold standard had an overall negative effect. After realignment, the concordance rates between Illumina and Affymetrix arrays dropped from 0.997 to 0.949 for normal and from 0.996 to 0.942 for tumour tissues. The effects were most pronounced for Bcftools, dropping from 0.865 to 0.735 for normal and from 0.898 to 0.734 for tumour tissues. For FaSD, changes were 0.882 to 0.864 for normal and 0.927 to 0.867 for tumour tissues. Both MAQ and SOAPsnp had better concordance 
rates with the Illumina array after realignment. For MAQ, changes were 0.397 to 0.439 for normal and 0.401 to 0.469 for tumour tissues, and for SOAPsnp, changes were 0.417 to 0.439 for normal and 0.409 to 0.472 for tumour tissues (Tables 1 and 6). We further evaluated the AUC of the different tools after realignment. We found that FaSD performed better than the other tools regardless of whether the data was pre-processed or not, or whether the Affymetrix or Illumina array benchmarks were used (Supplementary Tables S15-18).

We evaluated the performance of the different tools using the three criteria of concordance rate, AUC and non-reference concordance rate. These criteria were applied in different situations (Supplementary Table S1). When one of the two callers is used as the benchmark, the concordance rate is used to measure the proportion of correctly called $\mathrm{AA}, \mathrm{AB}$ and $\mathrm{BB}$ genotypes in the results. The SNP caller must use a specific cutoff for genotype calls, so the concordance will only be valid under this cutoff. In contrast, AUC measures the overall performance of two classification groups, AA and non-AA (non-SNP and SNP). It is a comprehensive measurement because it is not limited to a specific cutoff, but it does not distinguish between $\mathrm{AB}$ and $\mathrm{BB}$. Therefore, the concordance and AUC measurements may not always be consistent. For example, Bcftools has high concordance rates (Table 1 and Supplementary Table S2) but poor AUC (Supplementary Tables S3 and S4). When a program is applied on genome-wide data, the SNP calling result will be highly unbalanced because the chance of calling a SNP is less than $1 \%$ (predominately AA). Under this situation, the non-reference concordance rate is more appropriate than concordance rate for performance assessment, because it focus on measuring the quality of called $\mathrm{AB}$ and $\mathrm{BB}$ genotypes.

FaSD can be used as good complementary program to either GATK or Bcftools. The accuracy rate for SNPs called by both FaSD and Bcftools was the highest at $99.9 \%$ (Fig. 2). For SNPs called by both FaSD and GATK, the rate was also high at $99.7 \%$. In contrast, the accuracy is far less for SNPs called by any other two programs. For example, SNPs called by both FaSD and MAQ (but not by others) had an accuracy rate of $65.6 \%$, and for GATK and Bcftools the accuracy was $46.2 \%$. SNPs called individually by FaSD, GATK, Bcftools and MAQ had accuracies of $63.3 \%, 43.3 \%$, $35.9 \%$ and $2.0 \%$, respectively. We further investigated SNP loci called uniquely by FaSD and confirmed by both Affymetrix and Illumina SNP arrays (some examples are shown in Supplementary Figs S3-S5). In general, FaSD made accurate SNP calls even at loci with low-depth reads, with intermediate sequencing quality, and at the two ends of the read, compared with other programs that failed to make SNP calls. In summary, we recommend using both FaSD with either GATK or Bcftools for SNP calls. If the user requires a larger number of SNP calls, we suggest using FaSD on its own or FaSD and MAQ combined. The next best option would be to use GATK on its own or GATK and Bcftools combined.

The FaSD program was implemented in $\mathrm{C}^{++}$, which can easily be compiled to run on all platforms. Once the result of alignment is obtained, our model is able to detect SNP sites with high speed based on the pileup files. The compiled programs for Linux or Windows and demonstration data can be downloaded freely at http://jjwanglab.org/FaSD.

\section{Methods}

Data sets. We used NGS data from both a blood-derived normal sample and GBM tumour sample sequenced in the TCGA project. The reads (Sequence Read Archive (SRA) accession code: SRX006325) of the blood-derived normal sample (TCGA accession code: TCGA-06-0188-10B-01D-0373-08) were from a male with untreated GBM (TCGA accession code: TCGA-06-0188). The reads (SRX006310) of the GBM tumour sample were from primary tumour tissue (TCGA-06-018801A-01D-0373-08) from the same male with untreated GBM (TCGA-06-0188) ${ }^{19}$.
Both the samples were sequenced on the Illumina Genome Analyzer II platform: the normal sample was prepared by $2 \times 76$ bp paired-end library construction (Solexa-8304) and the GBM sample was prepared by $2 \times 76$ bp paired-end library construction (Solexa-8303). To evaluate NGS data from the SOLiD platform, reads (SRX015368) from the primary tumour tissue (TCGA-13-0720-01A-01D-0445-10) of a female OV patient (TCGA-13-0720) were used ( $\sim 6$-fold). The sequences, all in fastq format (csfastq for SOLiD), were extracted from the NCBI database of genotype and phenotype (dbGap) using the SRA toolkit. The raw data obtained using SRA was not filtered or modified (besides trimming). We merged the results from several runs to reach 30 gigabases at $10 \times$ coverage for each data set. These data sets were also genotyped using Illumina humanhap550 genotyping beadchip array and Affymetrix genome-wide human SNP array 6.0. The genotype data were downloaded from TCGA portal and used as our gold standards. To test the SNP callers' performance without using SNP arrays as the benchmark, we used data from one Yoruba individual (1,000 genomes accession code: NA19240) with high coverage MAQ alignment data of chromosome $21(39 \times)$ and $22(40 \times)$ generated by the pilot 2 phase of 1,000 Genomes Project ${ }^{28}$. To compare the performance of FaSD on pooled samples with the performance of GATK and Bcftools, we used the publically available sequencing data from $40 \mathrm{CEU}$ individuals in the pilot 1 phase of 1,000 Genomes Project. All 40 individuals were sequenced to $\sim 4 \times$ coverage genome-wide on a variety of platforms and from a variety of sequencing centres ${ }^{29}$. Sequencing data on chromosome 21 of a CEU trio (NA12878, NA12891 and NA12892) out of the 40 individuals was chosen as our evaluation subjects. This trio was also sequenced to $\sim 30 \times$ coverage genome-wide in the pilot 2 phase of 1,000 Genomes Project. We used MAQ's SNPs calling result on chromosome 21 of this high-depth trio, NA12878 (37 $\times$, rounded sequencing depth of chromosome 21$)$, NA12891 $(36 \times$, rounded $)$ and NA12892 $(30 \times$, rounded $)$ as the evaluation benchmark.

The FaSD model. After obtaining alignments from pileup file, we used FaSD to call SNPs for each aligned position. A FaSD_score was used to measure the polymorphism probability that a certain locus is a SNP location and to determine its corresponding genotype. If the FaSD_score was greater than the cutoff score, we called the locus a SNP and gave its corresponding genotype. The FaSD_score was calculated using the alternative_score and the geometric mean of a mutation probability of reads (equation 1):

$$
\text { FaSD_Score }=- \text { alternative_score } \times \frac{\sum_{i=1}^{\text {Depth }} \log _{2}\left(P_{\left(\text {read }_{i} / \mathrm{ref}\right)}\right)}{\text { Depth }}
$$

The alternative_score was calculated according to equation 2 . The $\left(P_{\left(\mathrm{read}_{i} / \mathrm{ref}_{30}\right)}\right)$ is the probability of getting a read genotype when the reference allele is $\mathrm{known}^{30}$. At each position, there could be three possible genotypes: $\mathrm{AA}, \mathrm{AB}$ and $\mathrm{BB}$. $\mathrm{AA}$ is homozygous and matches the reference allele, $\mathrm{AB}$ is heterozygous and $\mathrm{BB}$ is homozygous but does not match the reference allele. For positions with depth $N, n$ reads will match the reference and the other $N-n$ reads will not match the reference. We assumed that the number of reads matching and not matching the reference will follow binomial distributions. We then calculated the probability of the observed read frequency for each of the three possible genotypes. If the genotype is $\mathrm{AA}$, then the probability of not matching the reference should be very low (to be consistent with the error rate in the mutation probability formula $\left(P_{\left(\mathrm{read}_{\mathrm{i}} / \mathrm{ref}\right)}\right)$ we set this to 0.001$)$, and thus the probability of matching the reference should be very high (we set this to be $1-0.001=0.999$ ). If the genotype is $A B$, the probability of matching the reference and non-reference should be equal (both set to 0.500$)$. Similarly, if the genotype is $\mathrm{BB}$, the probability of matching the reference should be 0.001 and of matching the non-reference should be 0.999 (equation 2). We then used the probability mass function of binomial distributions to calculate the joint probability of all $N$ reads. We then compared the joint probabilities from all three possible genotypes. The genotype with the highest probability was selected and the alternative_score was assigned (equation 2). The three different binomial distributions corresponding to $\mathrm{AA}, \mathrm{AB}$ and $\mathrm{BB}$ were assigned the $P$ parameter $0.001,0.500$ and 0.999 , respectively. We then checked whether these parameters fitted our data. Taking into account the possible flaws in the sequencing, alignment or construction of the data sets, the parameters for the three binomial distributions appeared to be acceptable. The assigned alternative_score of 0,1 and 2 represents the number of loci that are different from the reference allele for two allele loci. Positions with genotype $\mathrm{AB}$ or $\mathrm{BB}$ are considered as SNP locations.

$$
\text { Alternative_Score }=\left\{\begin{array}{l}
0+\text { pseudo_score, } \operatorname{when}\left(\begin{array}{l}
N \\
m
\end{array}\right)(0.999)^{m}(0.001)^{N-m} \text { is } \max \\
1+\text { pseudo_score, } \operatorname{when}\left(\begin{array}{l}
N \\
m
\end{array}\right)(0.500)^{m}(0.500)^{N-m} \text { is } \max \\
2+\text { pseudo_score, } \operatorname{when}\left(\begin{array}{l}
N \\
m
\end{array}\right)(0.001)^{m}(0.999)^{N-m} \text { is max }
\end{array}\right.
$$

$N$ is the depth of the reads, and $m$ is the occurrence of reference allele at the position. We added a pseudo_score to avoid an alternative_score of 0 . By default, we set the pseudo_score to 0.01 . The alternative_score depends on which one of the three possible genotypes/models (reference homozygote, non-reference

homozygote, and heterozygote) explain the data the best. For example, if the locus 
has a total of five reads, with three Gs and two Cs, and the corresponding reference is $\mathrm{G}$ : the probability of getting the above reads with the reference homozygote model is $\left(\begin{array}{c}N \\ m\end{array}\right)(0.999)^{m}(0.001)^{N-m}=\left(\begin{array}{l}5 \\ 3\end{array}\right)(0.999)^{3}(0.001)^{5-3}=9.9 \times 10^{-6}$; the probability for the heterozygote model is $\left(\begin{array}{l}N \\ m\end{array}\right)(0.500)^{m}(0.500)^{N-m}=\left(\begin{array}{l}5 \\ 3\end{array}\right)(0.500)^{3}$ $(0.500)^{5-3}=0.31$; the probability for the non-reference homozygote model is $\left(\begin{array}{l}N \\ m\end{array}\right)(0.001)^{m}(0.999)^{N-m}=\left(\begin{array}{l}5 \\ 3\end{array}\right)(0.001)^{3}(0.999)^{5-3}=9.9 \times 10^{-9}$. We choose the heterozygote model because it has the highest probability of 0.31 , and we assign an alternative_score $=1+0.01=1.01$. Using equation 1, we obtain a FaSD_score of 5.07 , which is between our default cutoffs of 3.2 and 15.8, so we assign heterozygous genotype GC to this locus.

The estimated SNP rate between two distinct human haploid chromosomes has been reported to be close to 0.001 (ref. 30), and transitions are almost four times more frequent than transversions among substitutions ${ }^{31}$. The different frequencies of transitions and transversions mean they would have different contributions to the SNP calling. To discriminate these contributions, we integrated a $\left(P_{\left(\text {read }_{i} / \text { ref }\right)}\right)$ for each read into our final FaSD_score. We calculated the SNP rate and transition/transversion ratio in our GBM tumour and normal data sets, and found similar ratios (data not shown). Therefore, we used the above reported values to calculate $\left(P_{\left(\text {read }_{i} / \text { ref }_{)}\right)}\right)$(Supplementary Table S19). For each read, we compared the base at current loci with the reference allele to obtain a $\left(P_{\left(\text {read }_{i} / \text { ref }\right)}\right)$. From this table, we calculated the geometric mean of the mutation probability using equation 1 . We incorporated the effects of transitions and transversions into our FaSD model. The average of log-odd $\left(P_{(\text {read } / \text { ref })}\right)$ of all the $N$ reads was obtained, and the product of this value with the alternative_score was combined to form a FaSD_score, which was used to call SNPs. The higher the FaSD_score, the more likely a site is a SNP position. The default cutoffs of FaSD are determined mathematically. Because different types of errors such as sequencing or mapping errors during NGS could raise the FaSD score of a nonSNP site, we suggest the use of a cutoff value higher than the default to remove false positives, especially with low quality sequencing data. A user interface for setting the user-specific cutoff has been implemented in our FaSD program. The model has the ability to handle both the individual data sets and the pooled data sets.

Performance evaluation using SNP arrays. We used Illumina and Affymetrix SNP arrays as gold standards to evaluate the performance of FaSD and other tools. We excluded all sites whose depths were lower than 4 . The quality score is absent for the Illumina HumanHap550 Genotyping BeadChip data, so we accepted all the genotype entries. The Affymetrix genome-wide human SNP array 6.0 provides confidence scores for genotype quality using the Birdseed algorithm. Here, we chose the high-quality SNP probes as our test data set

by removing the probes with confidence scores above 0.018 .

For SOAPsnp and MAQ, we assigned the Phred-scaled probability that the genotype is identical to the reference, the so called 'SNP quality' as the predictor. There are several possibilities for the called genotypes: the reference homozygote, the non-reference homozygote, heterozygote and others. For the ROC curve and AUC calculations, we assigned the reference homozygote genotype as 0 , and all other genotypes as 1 . SNVmix2 outputs three genotypes, namely homozygous to reference $(\mathrm{AA})$, heterozygous genotype $(\mathrm{AB})$ and homozygous to the non-reference (BB). We considered $\mathrm{AB}$ and $\mathrm{BB}$ genotypes as a SNP, we added the probabilities of these two genotypes (AB and $\mathrm{BB}$ ) together to get the 'SNP probability' as the predicator. The GATK's UnifiedGenotyper and Bcftools generate SNP calls in the VCF format; the QUAL column is the 'SNP quality' and can be used as our predicator. For all callers mentioned above, the different values of predictor were used to draw the ROC curve, whereas the FaSD_score was used for FaSD. To test the stability of each software, we performed 1,000 times' bootstrap and obtained 1,000 AUC for each software. We then used Wilcoxon test to determine whether FaSD performed better than the other tools.

The genotype concordance and non-reference concordance. To measure the reliability of the different software/platforms for genotypes called from all SNPs (not limited to SNPs in Illumina and Affymetrix arrays), we determined genotype concordances among tools. Before the calculation of concordance, we restricted the tested loci to be the loci which has been genotyped in benchmark and whose depth were higher than 3 in the test alignment file to facilitate the reliability of the concordance result. Because GATK and Bcftools only report non-reference sites, we assigned genotype AA, the reference homozygous, to the loci which are not listed in SNP calling result of the above two tools. The non-reference concordance is measured in the similar way but excludes the concordant AA genotype because they are usually huge in number and easily detectable, but will greatly influence the measurement.

\section{References}

1. Ng, P. C. \& Henikoff, S. Predicting the effects of amino acid substitutions on protein function. Annu. Rev. Genomics Hum. Genet. 7, 61-80 (2006).

2. Kim, B. C. et al. SNP@Promoter: a database of human SNPs (single nucleotide polymorphisms) within the putative promoter regions. BMC Bioinformatics 9(Suppl 1): S2 (2008).

3. Yang, J. O., Kim, W. Y. \& Bhak, J. ssSNPTarget: genome-wide splice-site single nucleotide polymorphism database. Hum. Mutat. 30, E1010-E1020 (2009).
4. Hariharan, M., Scaria, V. \& Brahmachari, S. K. dbSMR: a novel resource of genome-wide SNPs affecting microRNA mediated regulation. BMC Bioinformatics 10, 108 (2009).

5. Venter, J. C. et al. The sequence of the human genome. Science 291, 1304-1351 (2001).

6. Lander, E. S. et al. Initial sequencing and analysis of the human genome. Nature 409, 860-921 (2001).

7. Frazer, K. A. et al. A second generation human haplotype map of over 3.1 million SNPs. Nature 449, 851-861 (2007).

8. Altshuler, D. et al. A haplotype map of the human genome. Nature 437, 1299-1320 (2005).

9. Shendure, J., Mitra, R. D., Varma, C. \& Church, G. M. Advanced sequencing technologies: methods and goals. Nat. Rev. Genet. 5, 335-344 (2004).

10. Wang, W. X., Wei, Z., Lam, T. W. \& Wang, J. W. Next generation sequencing has lower sequence coverage and poorer SNP-detection capability in the regulatory regions. Sci. Rep-UK 1, 55 (2011).

11. Morin, R. et al. Profiling the HeLa S3 transcriptome using randomly primed cDNA and massively parallel short-read sequencing. Biotechniques 45, 81-94 (2008).

12. Li, H., Ruan, J. \& Durbin, R. Mapping short DNA sequencing reads and calling variants using mapping quality scores. Genome Res. 18, 1851-1858 (2008).

13. Li, R. et al. SNP detection for massively parallel whole-genome resequencing. Genome Res. 19, 1124-1132 (2009).

14. Li, R., Li, Y., Kristiansen, K. \& Wang, J. SOAP: short oligonucleotide alignment program. Bioinformatics 24, 713-714 (2008).

15. Goya, R. et al. SNVMix: predicting single nucleotide variants from next-generation sequencing of tumors. Bioinformatics 26, 730-736 (2010).

16. Li, H. et al. The Sequence Alignment/Map format and SAMtools. Bioinformatics 25, 2078-2079 (2009).

17. McKenna, A. et al. The Genome Analysis Toolkit: a MapReduce framework for analyzing next-generation DNA sequencing data. Genome Res. 20, 1297-1303 (2010).

18. Malhis, N. \& Jones, S. J. High quality SNP calling using Illumina data at shallow coverage. Bioinformatics 26, 1029-1035 (2010).

19. Chin, L. et al. Comprehensive genomic characterization defines human glioblastoma genes and core pathways. Nature 455, 1061-1068 (2008).

20. Altshuler, D. L. et al. A map of human genome variation from population-scale sequencing. Nature 467, 1061-1073 (2010).

21. Hindorff, L. A. et al. Potential etiologic and functional implications of genome-wide association loci for human diseases and traits. Pro. Natl Acad. Sci. USA 106, 9362-9367 (2009).

22. Wei, Z., Wang, W., Hu, P. Z., Lyon, G. J. \& Hakonarson, H. SNVer: a statistical tool for variant calling in analysis of pooled or individual next-generation sequencing data. Nucleic Acids Res. 39, (2011).

23. DePristo, M. A. et al. A framework for variation discovery and genotyping using next-generation DNA sequencing data. Nat. Genet. 43, 491 (2011).

24. Nielsen, R., Paul, J. S., Albrechtsen, A. \& Song, Y. S. Genotype and SNP calling from next-generation sequencing data. Nat. Rev. Genet. 12, 443-451 (2011).

25. Browning, B. L. \& Yu, Z. X. simultaneous genotype calling and haplotype phase inference improves genotype accuracy and reduces false positive associations for genome-wide association studies. Genet. Epidemiol. 33, 783-783 (2009).

26. Visa, S. R. A. in IEEE Conference on Fuzzy Systems 749-754 (IEEE, 2005).

27. Weiss, G. M. \& Provost, F. Learning when training data are costly: the effect of class distribution on tree induction. J. Artif. Intell. Res. 19, 315-354 (2003).

28. Via, M., Gignoux, C. \& Burchard, E. G. The 1000 Genomes Project: new opportunities for research and social challenges. Genome Med. 2, 3 (2010).

29. Langmead, B., Trapnell, C., Pop, M. \& Salzberg, S. L. Ultrafast and memoryefficient alignment of short DNA sequences to the human genome. Genome Biol. 10, R25 (2009).

30. Sachidanandam, R. et al. A map of human genome sequence variation containing 1.42 million single nucleotide polymorphisms. Nature 409, 928-933 (2001).

31. Zhao, Z. \& Boerwinkle, E. Neighboring-nucleotide effects on single nucleotide polymorphisms: a study of 2.6 million polymorphisms across the human genome. Genome Res. 12, 1679-1686 (2002).

\section{Acknowledgements}

This work was supported by funding from the Research Grants Council (781511M, 778609M, N_HKU752/10, AoE M-04/04), Food and Health Bureau (10091262) of Hong Kong, NSFC of China (91229105) and the University of Hong Kong (10401206 and Genomic SRT).

\section{Author contributions}

Concept, design and method development (F.X., P.C.S. and J.W.); data preparation, pipeline design and performance evaluation (W.W., F.X. and J.W.); program implementation and algorithm optimization (F.X., P.W. and M.J.L.); and manuscript writing and editing (F.X., W.W., P.C.S. and J.W.). 


\section{Additional information}

Supplementary Information accompanies this paper on http://www.nature.com/ naturecommunications

Competing financial interests: The authors declare no competing financial interests.
Reprints and permission information is available online at http://npg.nature.com/ reprintsandpermissions/

How to cite this article: Xu, F. et al. A fast and accurate SNP detection algorithm for nextgeneration sequencing data. Nat. Commun. 3:1258 doi: 10.1038/ncomms2256 (2012). 